\title{
Impact of Gender and Working Environment on the Level of State Anxiety among Primary and Middle School Teachers in China
}

\author{
Lijuan Wang, Dajun Zhang \\ Center for Psychological Health Research, School of Psychology, Southwest University, Chongqing, China \\ Email:wlj0407@swu.edu.cn
}

Received October 28, 2011; revised November 29, 2011; accepted December 15, 2011

\begin{abstract}
State anxiety has become serious among primary and middle school teachers in China. By using stratified random sampling, we selected 2790 primary and middle school teachers as participants. They were from seven regions of China, among them 2278 were valid subjects. Constructed on the basis of anxiety content, the Teachers' State Anxiety Questionnaire (TSAQ) was used in the investigation. The result of the survey shows that primary and middle school teachers are generally affected by state anxiety; further analysis indicates that the prevalence of state anxiety is relatively higher among male teachers, teachers from key middle schools, teachers working more than 10 hours a day, and teachers loaded with additional task as head teachers. This is directly connected with China's national situation, teaching environment, discrepancies in teachers themselves, and their own ability of self-regulation.
\end{abstract}

Keywords: Primary and Middle School Teachers; State Anxiety; Prevalence

\section{Introduction}

State anxiety (S-AT) is generally related to negative events and stress. Although an occasional experience of temporary state anxiety causes no harm, long-term experiences of anxiety over time would affect the human body and mind seriously. Currently, primary and middle school teachers in China, faced with unprecedentedly higher working pressure and more emergencies in work, experience different levels of state anxiety more often. So, what is the general status of primary and middle school teachers' state anxiety? What kind of epidemiological difference can be caused by gender, work hours per day, and school type, etc.? What are the underlying causes of this situation? To find out the working status of primary and secondary teachers in China and to investigate state anxiety among them, these issues need to be clarified.

\section{Method}

The research goal of this thesis is to explore the epidemic-ological discrepancies of state anxiety experienced by primary and middle school teachers in China, due to the difference in gender, working hours, school types and grade levels, etc., and to propose some oriented prophylactico-therapeutic measures. Employing stratified random sampling and questionnaire survey, we selected 2790 teachers from key and general primary and middle schools in seven regions in China to be our subjects, with 2501 questionnaires back and the response rate being $89.64 \%$. With 223 invalid questionnaires excluded, we got an effective rate of $81.65 \%$ in response.

The self-compiled Teachers' State Anxiety Questionnaire (TSAQ), consisting of 30 questions, is employed to be our survey tool. There are five first-order factors in this questionnaire, namely Low agreement between the work and the reward, Low agreement between the selfdesired goals and the results, Conflict of professional self, Conflict between teacher role and family role, and Sense of control. The Cronbach coefficient $\alpha$ of the self-compiled TSAQ is 0.946 , indicating high internal consistency reliability. The result of confirmatory factor analysis (CFA) shows that the questionnaire has good construct validity. Compared with Zung's Self-rating Anxiety Scale, the Pearson correlation and Spearman correlation are 0.425 and 0.440. Compared with Spielberger's' State Anxiety Scale, the Pearson correlation and Spearman correlation are 0.433 and 0.447 . These comparisons show that TSAQ has good criterion validity.

In general, if a factor is distributed within the upper and lower standard deviation $(S D)$ of the norm group average $(M)$, it means that the state anxiety is median. If a factor is distributed above $M+S D$ or below $M-S D$, it suggests negligible state anxiety and serious state anxiety 
respectively. The average and standard deviation of primary and middle school teachers of different genders, types of school and grades related to each factor are presented in Table 1.

TSAQ differentiates from other state anxiety scale in the following two ways: in the structure of the questionnaire, most of the previous state anxiety scales count state anxiety to be one-dimensional, but TSAQ view it as a multi-dimensional structure; in the content of the questionnaire, most of the previous state anxiety scales focus on state anxiety symptoms, while TSAQ is compiled on the basis of the causes of state anxiety. This avoids the deficiency of the measurement tool, i.e., not being able to distinguish state anxiety from trait anxiety. It also proposes a way out of the insufficiency of unchangeability in state anxiety at different situations and for different groups, for it is previously viewed as one-dimensional. The four-point scoring system and the form of obligatory choice are employed in the specific implementation. SPSS16.0 software assists the final statistical analysis. By virtue of the uncertainty of normal distribution of the data, non-parametric test is adopted in analyzing the specific data, with the results being two-sided testable.

Table 1. Comparison of state anxiety among primary and middle school teachers in relation to different groups and factors.

\begin{tabular}{|c|c|c|c|c|c|c|c|}
\hline \multicolumn{2}{|c|}{ Groups } & \multirow{2}{*}{$\begin{array}{c}\text { TSSA } \\
2.43\end{array}$} & \multirow{2}{*}{$\begin{array}{c}\text { LAWR } \\
2.25\end{array}$} & \multirow{2}{*}{$\begin{array}{c}\text { LASGR } \\
2.44\end{array}$} & \multirow{2}{*}{$\begin{array}{c}\mathrm{SC} \\
2.39\end{array}$} & \multirow{2}{*}{$\begin{array}{l}\text { CPS } \\
2.55\end{array}$} & \multirow{2}{*}{$\begin{array}{c}\text { CTRFR } \\
2.70\end{array}$} \\
\hline male & $M$ & & & & & & \\
\hline & $S D$ & 0.58 & 0.62 & 0.66 & 0.65 & 0.73 & 0.72 \\
\hline \multirow[t]{2}{*}{ female } & $M$ & 2.32 & 2.11 & 2.35 & 2.28 & 2.46 & 2.60 \\
\hline & $S D$ & 0.61 & 0.64 & 0.66 & 0.68 & 0.77 & 0.76 \\
\hline \multirow[t]{2}{*}{ primary } & $M$ & 2.26 & 1.98 & 2.23 & 2.32 & 2.43 & 2.48 \\
\hline & $S D$ & 0.62 & 0.63 & 0.63 & 0.72 & 0.80 & 0.79 \\
\hline \multirow{2}{*}{$\begin{array}{l}\text { junior } \\
\text { high }\end{array}$} & $M$ & 2.44 & 2.25 & 2.46 & 2.40 & 2.54 & 2.74 \\
\hline & $S D$ & 0.60 & 0.63 & 0.65 & 0.66 & 0.75 & 0.75 \\
\hline \multirow{2}{*}{$\begin{array}{l}\text { senior } \\
\text { high }\end{array}$} & $M$ & 2.38 & 2.20 & 2.42 & 2.29 & 2.51 & 2.66 \\
\hline & $S D$ & 0.58 & 0.62 & 0.66 & 0.65 & 0.74 & 0.73 \\
\hline \multirow{2}{*}{$\begin{array}{l}\text { key } \\
\text { middle }\end{array}$} & $M$ & 2.43 & 2.23 & 2.44 & 2.34 & 2.58 & 2.73 \\
\hline & $S D$ & 0.59 & 0.63 & 0.65 & 0.66 & 0.74 & 0.73 \\
\hline \multirow{2}{*}{$\begin{array}{l}\text { general } \\
\text { middle }\end{array}$} & $M$ & 2.28 & 2.14 & 2.40 & 2.29 & 2.28 & 2.49 \\
\hline & $S D$ & 0.57 & 0.62 & 0.66 & 0.64 & 0.72 & 0.73 \\
\hline \multirow[t]{2}{*}{ totality } & $M$ & 2.37 & 2.16 & 2.39 & 2.33 & 2.50 & 2.64 \\
\hline & $S D$ & 0.60 & 0.63 & 0.66 & 0.67 & 0.76 & 0.75 \\
\hline
\end{tabular}

TSSA:Total score of state anxiety; LAWR:Low agreement between the work and the reward; LASGR:Low agreement between the self-desired goals and the results; SC:Sense of control ; CPS:Conflict of professional self; CTRFR: Conflict between teacher role and family role.

\section{Results}

\subsection{Prevalence of State Anxiety among Primary and Middle School Teachers}

The average $\mathrm{M}$, standard deviation $S D$ and critical value $M \pm S D$ of the totality of primary and middle school teachers' state anxiety are $2.37,0.60$ and $1.77-2.97$ respectively. Further data analysis shows that the number of teachers above $2.97(M+S D)$ are 409 altogether, accounting for $17.95 \%$; the number of teachers below 1.77 $(M-S D)$ is 393 altogether, accounting for $17.25 \%$. In other words, $17.95 \%$ of primary and middle school teachers experience strong sense of state anxiety in teaching and $17.25 \%$ seldom experience state anxiety, while $64.80 \%$ experience medium state anxiety. Non-parametric test of multiple independent samples shows: $\chi^{2}=1380.70, p<$ 0.001 , suggesting that the prevalence of medium, severe, and negligible state anxiety are significantly different.

\subsection{State Anxiety among Male and Female Teachers from Primary and Middle Schools}

Of all 895 male subjects, average scores of 121 teachers are below 1.77, accounting for $13.52 \%$; average scores of 157 teachers are above 2.97 , accounting for $17.54 \%$; the other $68.94 \%$ suffer medium state anxiety. Among all the 1383 female subjects, there are 271 whose average score is less than 1.77 , accounting for $19.60 \%$, and 252 whose average score is above 2.97, accounting for $18.22 \%$; while the other $62.18 \%$ suffer medium state anxiety. The non-parametric testing of two independent samples shows: $Z=-4.34, p<0.00$, indicating significant difference of the prevalence of state anxiety between male and female teachers.

Further analysis shows that state anxiety level of primary and secondary teachers of different genders is different in the distribution pattern: the number of male teachers with less obvious symptoms is smaller than that of female counterparts; male teachers with medium level of symptoms are more than their female counterparts; female teachers with intensive symptoms are a little more than their male counterparts.

\subsection{State Anxiety among Teachers from Different Types of Primary and Middle Schools}

The average scores of 186 teachers among all the 1403 subjects from key middle schools are below 1.77, accounting for $13.26 \%$; the average scores of 276 teachers are above 2.97, accounting for $19.67 \%$; while the other $67.07 \%$ suffer medium state anxiety. Of 370 regular middle school teachers being surveyed, there are 82 whose average score is less than 1.77 , accounting for $22.16 \%$; 50 whose average score is more than 2.97 , accounting for 
$13.51 \%$; while the rest $64.33 \%$ suffer medium state anxiety. The survey on 505 primary school teachers shows that the average score of 123 people is below 1.77 , accounting for $24.36 \%$; the average score of 82 is above 2.97 , accounting for $16.23 \%$; while $59.41 \%$ of the primary school teachers surveyed suffer from medium state anxiety. By comparing these three groups and non-parametric testing multiple independent samples, we find that: $\chi^{2}=34.78, p<0.001$, indicating that the prevalence of state anxiety between teachers in key middle schools, regular middle schools and primary schools are significantly different.

Further analysis demonstrates that there are significant discrepancies in the distribution of population at different state anxiety levels, namely negligible, medium and serious levels, among teachers in key middle schools, regular middle schools and primary schools. The lowest proportion of teachers who only suffer from negligible symptoms occurs in the key middle school group, while the population proportion suffering from medium and severe symptoms is the highest among three groups. The lowest population proportion of suffering severe symptoms occurs in the regular middle school group. The proportion of people who only suffer negligible symptoms is lowest in the primary school group.

\subsection{State Anxiety of Primary and Secondary Teachers at Different Grade Levels}

The survey of 1211 senior high school teachers shows that the average scores of 193 subjects are below 1.77, which accounts for $15.94 \%$; those of 203 subjects are above 2.97 , accounting for $16.76 \%$; while the other $67.30 \%$ suffer from medium state anxiety. Among 562 junior high teachers being surveyed, average scores of 74 teachers is less than 1.77 , accounting for $13.16 \%$; average scores of 123 teachers are more than 2.97, accounting for $21.89 \%$; while the remaining $64.95 \%$ have moderate state anxiety. 505 primary school teachers have been surveyed, among whom 123 teachers receive average scores below 1.77 which accounts for $24.36 \%$; 82 teachers receive average scores above 2.97 which accounts for $16.23 \%$; and the $59.41 \%$ teachers left suffer from medium state anxiety. After comparing the three groups with non-parametric test on multiple independent samples, we find that: $\chi^{2}=22.24, p<0.001$, indicating significant differences in prevalence of state anxiety among senior high, junior high and primary school teachers.

Further analysis reveals that there are prominent proportion differences at different state anxiety levels between elementary, middle and high school teachers. The primary school group is found to include the highest percentage of teachers who only suffer from negligible state anxiety and the lowest percentage of those who have moderate and severe symptoms. The junior high group is found to have the highest percentage of severe state anxiety and the lowest percentage of negligible state anxiety. The highest percentage of medium state anxiety occurs in the senior high group.

\subsection{State Anxiety of Primary and Middle School Teachers with Different Working Hours}

Through the survey we find that there are discrepancies in the length of teachers' daily working time, with some working less than 8 hours per day, while others working far more than 8 hours a day. Then, what kind of correlation exists between the duration of teachers' daily work and the prevalence of their state anxiety? Here the length of working hours are divided into eight sections: 8 hours or less, 9 hours, 10 hours, 11 hours, 12 hours, 13 hours, 14 hours, and 15 hours or more. The comparison between eight groups with non-parametric testing multiple independent samples shows: $\chi^{2}=180.54, p<0.001$, which reveals that, due to the difference in those primary and middle school teachers' daily working time, the prevalence of their state anxiety are significantly different (see Figure 1).

The result shows that the percentage of those who seldom suffer from state anxiety is lowest in the eighthour-and-less group. 10 working hours daily seems to be a dividing line, from which the population percentage at different levels starting to change dramatically. When the daily working hours are less than 10 , the percentage of those who only suffer from negligible symptoms is highest while that of severe symptoms is lowest; when the daily working hours are more than 10 , the latter turns into the highest. Therefore, the length of daily working time plays a very important role in deciding the level of state anxiety among teachers.

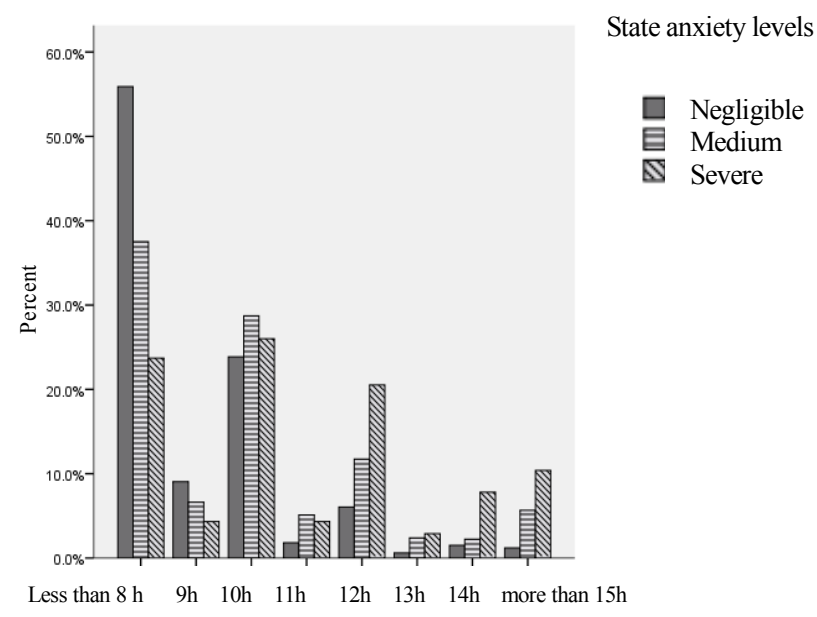

Figure 1. Comparison of prevalence of state anxiety at each level among primary and middle school teachers with different daily working hours. 


\subsection{State Anxiety of Primary and Middle School Teachers with Different Working Load}

Some teachers need to do nothing but teaching, while others, apart from teaching, are undertaking the responsibility of head teachers. There are 944 teachers with double duties in this investigation, $11.86 \%$ of which belongs to the group with the average score below 1.77 and $22.03 \%$ of which falls into the group with the average score above 2.97 . The $66.11 \%$ left suffer from medium state anxiety. The population of teachers assuming teaching as their only responsibility is 1334 , among which people fall into the three groups with average scores below 1.77, above 2.97, and between 1.77 and 2.91 (entitling moderate state anxiety), accounting for $20.84 \%$, $15.14 \%$ and 64.02 respectively. Through non-parametric tests on these two independent samples, we find that: $Z=$ $-7.80, p<0.001$, which means dramatic discrepancies in state anxiety levels of those teachers loaded with extra work.

The percentage of teachers suffering from severe state anxiety is very high among teachers with dual responsibilities, and the percentage of teachers suffering from negligible state anxiety is quite low. By contrast, the proportion of teachers suffering from severe state anxiety is fairly low and that of teachers suffer from negligible state anxiety is fairly high among teachers only loaded with teaching. Therefore, the workload plays an influential role in level of state anxiety among teachers.

\section{Discussions}

\subsection{Prevalence of State Anxiety among Primary and Middle School Teachers}

There are few previous researches on the issue of state anxiety prevalence among primary and middle school teachers. One of those few researches, for example, conducted by Li Zuojia and others [1], in which self-rating anxiety scale (SAS) and symptom check list-90 (SCL-90) were employed, showed that only $5.91 \%$ of primary and middle school teachers suffer from anxiety disorders, which is considerably lower than the prevalence among those involved in other work areas. They contributed this discrepancy to the relatively stronger sense of satisfaction enjoyed by teachers than that enjoyed by people with other occupations. Our results show that, the prevalence of state anxiety among primary and middle school teachers is $82.75 \%$, with the prevalence of severe and medium suffering being $17.95 \%$ and $64.80 \%$ respectively. This discrepancy is mainly due to the different measuring tools.

The short duration of state anxiety determine that the measuring of its symptoms cannot to be deferred. The direct usage of "self-rating anxiety scale" or "state anxiety scale", whose measuring both are based on symptoms in those previous studies, excluded a lot of individuals from the state anxiety group, especially those whose anxiety does not reach the level of anxiety disorder or has vanished by the time. Since TSAQ mainly focuses on evaluating the causes of their state anxiety, its results are able to reflect the real state of their state anxiety. Of course, the resulting state anxiety prevalence being $82.75 \%$ in this study does not mean that $82.75 \%$ of teachers suffer from medium or even severe anxiety disorders, but indicates that there are $82.75 \%$ of the teachers who are regularly caught by medium or severe state anxiety in their work. Such a high prevalence also illustrates that there are many triggers for state anxiety in those teachers, which is composed of lack of control over teaching events, low self-desirability between commitment and reward, low self-desirability between the goal and result, strong self-conflict of career, and severe conflict between roles in school and in family, etc. Psychological researches have found that state anxiety and depression caused by long-standing dealing with pressure would render the organism more susceptible to many physical diseases, such as severe ulcers, heart disease, decline of immune system, and shortening of life expectance [2]. Moreover, severe anxiety emotions are likely to lower the victim's living condition and work efficiency, which in turn would mess up his or her life and work. Thus, it is a priority to take effective measures to relieve primary and middle school teachers in China from state anxiety.

\subsection{Discrepancies in State Anxiety Caused by Tasks and Working Hours}

As mentioned earlier, the prevalence of state anxiety among teachers who, besides teaching, act as head teachers at the same time is notably higher than that among teachers undertaking teaching only. Undertaking two duties forces those teachers to spend more time and energy to fulfill the extra task. To do this is often at the expense of their personal sphere for relaxation, exercising, entertainment, and even executing family obligations. As the over-commitment does not get the expected return, the state anxiety will most probably occur. On the other hand, the level of state anxiety clearly correlates with the length of daily working hours. As we have seen, the proportion of teachers who are disturbed by medium or severe state anxiety goes higher when their daily working time has to be lengthened. It is a fact that among those 2278 primary and middle school teachers, only $38.15 \%$ of whom do not have to work longer than 8 hours a day, which means that as many as $61.95 \%$ of them work more than 8 hours a day. Therefore, it is clear that the urgent need for institution in charge of education can help alleviate teachers' workload. Teachers also need to respond actively to meet the increasing need of higher work efficiency and healthier personal psychology (such as, by 
doing physical exercises, developing interests and hobbies, listening to soothing music more often, communicating regularly with friends or family members, taking psychological counseling, etc.).

Gender, Grade level (elementary, junior and senior high), and School type (general and key) are also prominent factors in influencing the state anxiety prevalence among teachers. Compared with female teachers, medium state anxiety prevalence among male teachers is remarkably higher, but the prevalence of severe state anxiety is slightly lower. First of all, from the perspective of individual achievement motivation, there is a big difference between men and women. King (1995) [3] pointes out that in China, compared with women, men have higher expectations of achievements, stronger sense of competition and achievement, harsher self-evaluation and higher autonomy on achievement, all of which lead men at work to be more inclined to participate in competition and take extra tasks, resulting in a higher baseline level, so as to giving rise to level of higher state anxiety. Secondly, in seeking social support, men and women also vary considerably. It was found that when faced with pressure, women are more likely to seek the support from others (Tamres 2002) [4]. Another group of researchers including Zhou Shaoping [5], through a investigation conducted among some elementary and middle school teachers in the city of Chenzhou, also found that male teachers get lower scores than their female counterparts in fields of social relations and environment, overall living quality and support seeking. Last but not the least, men and women also vary dramatically in spending time to expressing feelings. Many studies have found that women tend to spend more time on the phone [6]. Even in this electronic information age, Women's eagerness of seeking relief does not weaken. It was found that women are more often to use computers to write e-males, rather than to play games, as men would often do (Crabtree 2002) [7]. Many of those teach- ing events are stressful to primary and middle school tea- chers, in dealing with these pressures, female teachers would be inclined to ask for advice or get someone to talk about it, while male teachers tend to choose bearing it alone. Therefore, the prevalence of moderate state anxiety among female teachers is significantly lower than that among male teachers. However, being in such a full schedule with no time left for seeking help or talking with others, female teachers are more vulnerable to be depressed by anxiety and depression [8]. This explains the higher prevalence of severe state anxiety among female teachers than that among the male counterparts. People are more likely to seek social support from female friends [9]. In view of this, the proposal for education administrations is to create a more harmonious and healthy interpersonal environment, and more attention should be paid to male teachers' marital happiness and family harmony, so as to decrease state anxiety by improving the interpersonal surrounding.

Teachers, working at different grade levels face unequal challenges of preparing their students for higher education, undertake tasks of a different nature, and deal with students in different physiological and psychological problems. These are the main causes for the remarkable difference of state anxiety prevalence among elementary, junior and senior teachers. Teachers in senior high schools face the challenge of the university entrance examination, an annual event for the whole public, making them over-loaded both with work and pressure. This underlines the highest prevalence of state anxiety among senior high school teachers. As for junior school teachers, although they are not faced with such complicated and stressful work, their students have just stepped into adolescence. These teenagers are always the most rebellious ones. Even after the teacher devotes lots of time, energy and affection to some student, he or she may remain unchanged. On the other hand, junior school teachers are allowed by education administrations, society and legislative bodies with very limited resources to educate students, which leads many teachers frustrated and confused when teaching pupils. That is why the highest prevalence of severe state anxiety occurs among junior school teachers. In order to pursue effective approaches to relieve teachers from state anxiety, the load of work should be lightened, and the achievement should be evaluated in a more flexible way. How to offer teachers more means and resources should be considered before they can discipline pupils more appropriately and effectively.

Key middle schools are different from their general school counterparts both in competitive atmosphere and in school rules and regulations. Compared to general middle schools, key middle schools have more stringent rules and regulations, more fierce competitions, more intense work for their teachers. Fierce competitions and heavy work, would inevitably lead key middle school teachers quite vulnerable to be disturbed by state anxiety.

\section{Conclusions}

From the discussions and statistics above, the following two conclusions can be drawn: 1) there is high prevalence of state anxiety among primary and middle school teachers in China; 2) nature of task, daily working hours, gender, grade levels (elementary, junior and senior high), etc. are vital in influencing the state anxiety prevalence among teachers. In order to reduce the level of state anxiety among teachers, two aspects should be considered. First of all, their workload must be lightened. Secondly, they should be allowed with more means and resources to educate pupils. Besides, of course, social recognition 
and understanding, especially from education administrators as well as from students and their parents, would help a lot. Their state anxiety can also be relieved if they manage to develop themselves both physiologically and psychologically.

\section{Acknowledgements}

The present research is sponsored by Eleventh Five-year Project in Educational Sciences in Chongqing 2010 (no. 2010-GJ-0202), Research Program for Doctorates (no. SWU0909324), the Research Program on Strategies for Fostering Juveniles' Cognition by Chongqing Key Research Center for Arts and Humanities), and the Fundamental Research Funds for the Central Universities (SWU1009089).

\section{REFERENCES}

[1] Z. J. Li, D. F. Zhao, et al., "Studies in Elementary and Secondary Teachers' Anxiety Symptoms and Related Factors," Shandong Psychiatry, Vol. 11, No. 3, 1998, pp. 23-24.

[2] D. Myers, "Psychology," 7th Edition, People's Posts and Telecommunications Press, Beijing, 2006, pp. 461-469.

[3] H. B. King, "Study on Chinese People's Achievement Motivation Differences in Gender," Psychological Science,
Vol. 18, No. 2, 1995, pp. 180-182.

[4] L. K. Tamres, V. S. Helgeson and D. Janicki, "Sex Differences in Coping Behavior: A Meta-Analytic Review and an Examination of Relative Coping," Personality and Social Psychology Review, Vol. 6, No. 1, 2002, pp. 2-30. doi:10.1207/S15327957PSPR0601_1

[5] S. P. Zhou, Q. W. Liu, Y. Zhou, J. X. Gao, J. Li, X. Liang, et al., "Research on Primary and Middle School Teachers's Living Quality in the City of Chenzhou and the influencing Factors," Journal of Xiangnan College (Medical Sciences), Vol. 9, No. 4, 2007, pp. 1-4.

[6] Z. Smoreda and C. Licoppe, "Gender-Specific Use of the Domestic Telephone," Social Psychology Quarterly, Vol. 63, No. 3, 2000, pp. 238-252. doi:10.2307/2695871

[7] S. Crabtree, "Gender Roles Reflected in Teen Tech Use," Gallup Tuesday Briefing, 22 January 2002, (last checked 3 October 2009).

http://www.gallup.com/poll/5209/gender-roles-reflected-t een-tech-use.aspx

[8] C. A. Li and X. Zeng, "Middle-Aged Patients with Anxiety and Depression Troubled by Arrhythmias and Gender Differences," Guangdong Medicine, Vol. 31, No. 5, 2010, pp. 611-612.

[9] L. A. Sapadin, "Friendship and Gender: Perspectives of Professional Men and Women," Journal of Social and Personal Relationships, Vol. 5, 1988, pp. 387-403. doi:10.1177/0265407588054001 